\title{
Intellectual disability-cataracts-calcified pinnae-myopathy syndrome
}

INSERM

\section{Source}

INSERM. (1999). Orphanet: an online rare disease and orphan drug data base. Intellectual disability-cataracts-calcified pinnae-myopathy syndrome. ORPHA:3042

Intellectual disability-cataracts-calcified pinnae-myopathy syndrome is a rare, genetic intellectual disability syndrome characterized by macrocephaly, hypotonia, dysmorphic facial features (wide forehead, ptosis, downslanting palpebral fissures, enlarged and calcified external ears, large jaw), sparse body hair, tall stature, and intellectual disability. Hearing loss, insulin-resistant diabetes, and progressive distal muscle wasting (leading to joint contractures) have also been reported in adulthood. Rare manifestations include behavioral abnormalities (aggression and restlessness), hypothyroidism, cerebral calcification, ataxia, and peripheral neuropathy. 